\title{
Oncology
}

\section{Acknowledgement to the Reviewers}

The Editors and the members of the editorial board would like to thank the following individuals for their expert assistance in acting as reviewers for Oncology in the period of February 1, 2014 to July 31, 2014.

Muneer H. Abidi, Detroit, Mich., USA

Hee Kyung Ahn, Incheon, Korea

Cristianne Alexandre, Joao Pessoa, Brazil

Tomio Arai, Tokyo, Japan

Lodovico Balducci, Tampa, Fla., USA

Jana Barinoff, Frankfurt am Main, Germany

Wolf O. Bechstein, Frankfurt am Main, Germany

Yves Bécouarn, Bordeaux, France

Pierluigi Benedetti Panici, Rome, Italy

Robert S. Benjamin, Houston, Tex., USA

Al B. Benson, Chicago, Ill., USA

Ellin Berman, New York, N.Y., USA

Aashish D. Bhatt, Columbus, Ohio, USA

Idoia Bilbao-Meseguer, Barakaldo, Spain

Giorgio Bogani, Varese, Italy

Franc Bourdeaut, Paris, France

Andrea Cariati, Genoa, Italy

Alexandre Carpentier, Paris, France

Brian I. Carr, Castellana Grotte, Italy

Susan Chafe, Edmonton, Alta., Canada

Ann-Lii Cheng, Taipei, Taiwan

Minsig Choi, Detroit, Mich., USA

Vincent Chung, Duarte, Calif., USA

James F. Cleary, Madison, Wisc., USA

Giovanni Codacci-Pisanelli, Rome, Italy

Robert L. Coleman, Houston, Tex., USA

Romain Coriat, Paris, France

Allan Covens, Toronto, Ont., Canada

Antonio Cristaudo, Rome, Italy

Neesha Dhani, Toronto, Ont., Canada

Andreas Dinkel, Munich, Germany

Frede Donskov, Aarhus, Denmark

Stefan Duensing, Heidelberg, Germany

Janice P. Dutcher, Bronx, N.Y., USA

Matthias P.A. Ebert, Mannheim, Germany
F. Efficace, Rome, Italy

Wolfgang W. Eisterer, Innsbruck, Austria

Bassel F. El-Rayes, Atlanta, Ga., USA

Ralph Ermoian, Seattle, Wash., USA

Marwan G. Fakih, Duarte, Calif., USA

Nicola Fazio, Milan, Italy

Dorothea Fischer, Lübeck, Germany

Junji Furuse, Tokyo, Japan

Karen Gelmon, Vancouver, B.C., Canada

Andreas Gescher, Leicester, UK

Sarbani Ghosh-Laskar, Mumbai, India

Stefan Glück, Miami, Fla., USA

Margarida M. Gonçalo, Coimbra, Portugal

John Gosney, Liverpool, UK

Juan-José Grau, Barcelona, Spain

Stefano Greggi, Naples, Italy

Rob Grimer, Birmingham, UK

Carlo Guastoni, Milan, Italy

Michael Haas, Munich, Germany

Phillip Harter, Essen, Germany

Akito Hata, Kobe, Japan

Beata Holkova, Richmond, Va., USA

Akihiro Homma, Sapporo, Japan

David Ilson, New York, N.Y., USA

Chikashi Ishioka, Sendai, Japan

Takeshi Isobe, Izumo, Japan

Rolf Issels, Munich, Germany

Namiki Izumi, Tokyo, Japan

Chang Wook Jeong, Seoul, Korea

Bernd Kasper, Mannheim, Germany

Shunsuke Kato, Tokyo, Japan

Satoshi Kato, Morioka, Japan

Noriyuki Katsumata, Kawasaki, Japan

Yoshiaki Kawaguchi, Isehara, Japan

Eric Kim, Rochester, N.Y., USA 


\section{Oncology}

Hans-Georg Kopp, Tübingen, Germany Andreas Krieg, Düsseldorf, Germany Judith R. Kroep, Leiden, The Netherlands Diana E. Lake, New York, N.Y., USA Rolf Lamerz, Munich, Germany Soohyeon Lee, Seoul, Korea Jae Myun Lee, Seoul, Korea Edward Libby, Seattle, Wash., USA Karl Heinz Link, Wiesbaden, Germany Antonio Lopez-Beltran, Cordoba, Spain Manfred P. Lutz, Saarbrücken, Germany John S. Macdonald, Castine, Maine, USA Makoto Maemondo, Natori, Japan Gina Mantia-Smaldone, Philadelphia, Pa., USA Georg Maschmeyer, Potsdam, Germany Donald C. McMillan, Glasgow, UK Gracia Merino, Leon, Spain Marco Carlo Merlano, Cuneo, Italy Masaki Mori, Suita, Japan Manabu Morimoto, Yokohama, Japan Kei Muro, Nagoya, Japan Nagalakshmi Nadiminty, Sacramento, Calif., USA Shoji Natsugoe, Kagoshima, Japan Sung Hoon Noh, Seoul, Korea Charles L.M. Olweny, Kampala, Uganda Nobuhiko Oridate, Yokohama, Japan Naohide Oue, Hiroshima, Japan Antonio Passaro, Milan, Italy Nitesh Patel, New Brunswick, N.J., USA Sandro Pignata, Naples, Italy M. Radhakrishna Pillai, Trivandrum, India Aleix Prat, Barcelona, Spain Jean Luc Prétet, Besançon, France Sarah Prinsloo, Houston, Tex., USA Camilla Qvortrup, Odense, Denmark Jacques Robert, Bordeaux, France
Achim Rody, Lübeck, Germany Alonzo Ross, Worcester, Mass., USA Ingo B. Runnebaum, Jena, Germany Suzanne Russo, Mobile, Ala., USA James Rutka, Toronto, Ont., Canada Sho Saeki, Kumamoto City, Japan Bruno Sangro, Pamplona, Spain Florian Schütz, Heidelberg, Germany Rajni Sethi, San Francisco, Calif., USA Lin Shen, Beijing, China Susanne Singer, Mainz, Germany Anil K. Sood, Houston, Tex., USA Pierre-Jean Souquet, Pierre-Benite, France Joanna Szkandera, Graz, Austria Makoto Tahara, Ciba, Japan Tetsuo Takehara, Osaka, Japan Hideo Takeshima, Miyazaki, Japan Motohiro Tamiya, Habikino, Japan Iain Tan, Singapore, Singapore Kyuichi Tanikawa, Kurume, Japan Mary Thomas, Palo Alto, Calif., USA Yasushi Toh, Fukuoka, Japan Saad Z. Usmani, Charlotte, N.C., USA Jan B. Vermorken, Edegem, Belgium Jaques J. Waisberg, Santo André, Brazil Martin Weber, Mainz, Germany Andrew Westwell, Cardiff, UK Takayuki Yakushijin, Osaka, Japan Takushi Yasuda, Osaka, Japan Wataru Yasui, Hiroshima, Japan Marc Ychou, Montpellier, France Cheol Yong Yoon, Seoul, Korea Wei Zhang, Chicago, Ill., USA Zhong-Zheng Zhu, Ningbo, China Jay-Jiguang Zhu, Houston, Tex., USA Marcin Zieliski, Zakopane, Poland 